\title{
Last Reflections of the Editor
}

\author{
Richard Staelin \\ Fuqua School of Business, Duke University, Durham, North Carolina 27708-0120 \\ richard.staelin@duke.edu
}

As I write this editorial, it was just about 47 months ago that I took over as editor-in-chief of Marketing Science. Over that period of time, I handled 585 manuscripts in one stage or another of the review process. (I still have responsibility for approximately 12 papers, plus another 20, which are associated with the special issue on Managerial Decision Making.) During the 38 months that I was the editor, the journal received 410 new submissions, including those for the special issue. To date, I accepted 59 of these papers, while the average turnaround for a paper was just over 75 days.

What did I learn from this experience, besides the fact that carrying home manuscripts every night can lead to a bad back? First of all, I have a much deeper appreciation for the complexity of the review process. One would like to think that the process is always reliable and valid, as well as constructive and speedy. As an editor, I felt I had only marginal control over the first two attributes, and then mainly by carefully selecting the review team. Where I felt I had the most impact was maintaining a "speedy" review process. In hindsight, almost all long review processes were directly linked to one slow evaluator. What I should have done to get around this problem was to process a paper within some cutoff date with whatever information was available at that time. For one reason or another, I had difficulty doing this and instead hoped (often to no avail) that the tardy individual would eventually respond and I (and the authors) would gain valuable new information.

I am less sanguine about influencing the reliability and validity of the reviews. It is probably true that we publish very little that is truly wrong and/or not interesting to a significant segment of our readers. Thus, the review process is reasonably good at screening out papers with technical problems and marginal contribution. That we catch most mistakes is not all that surprising when you realize that reviewers have been "trained" to look for errors and that only one of the (4) evaluators used for each paper has to catch a "fatal" flaw for the paper to be rejected. What is of more concern to me is the other type of error, i.e., reviewers suggesting rejection for a paper that actually should be published (albeit after some revisions).

\section{The Problem}

This raises a series of questions. What is the correct definition of a paper that should be published? Why are so many papers screened out by reviewers and what, if anything, can an editor do to rectify the situation?

Defining the characteristics of a paper that should be published is very difficult unless one uses abstract terms such as "relevant" or "makes a significant new contribution". Still, we all have seen papers that have changed the way we look at a field. Interestingly, there is strong empirical evidence that many of these papers did not have an easy time in the review process. For example, Akerof's paper "A Market for 'Lemons'" (1970) was initially rejected by three leading economics journals. Bob Lucas's 1972 paper introducing rational expectations concepts into macroeconomics and the 1973 paper by Fischer Black and Myron Scholes introducing their option pricing formula are other examples of papers with huge impacts that were originally rejected (Gans and Sheppard 1994). For more examples of classic papers that were rejected on first submission, see Campanario (1995). One might explain this by noting that these papers represent new ideas and challenge existing paradigms. They also often require the evaluator to think about the problem in a new way. Finally, because they are breaking new ground, there may initially be some "errors" in the author's logic, or presentation. Each of these characteristics can lead reviewers to suggest rejection.

If "classic" papers have a tough time in the review process, what about the solid paper that deserves to be published but may lack the "home-run" quality mentioned above? More generally, how reliable are review- 
ers in rating the quality of a paper? The answer is they are not very reliable. John Lynch (1998) in his ACR presidential address provides numerous references to studies that document the lack of interjudge reliability of the reviewers' overall recommendations. My personal experience leads me to believe this is particularly true for papers that are good (i.e., potentially publishable) but not great. (Said differently, the greatest convergence of opinion across evaluators seemed to occur when the paper clearly was flawed and/or made no significant contributions.)

What then is an editor to do when faced with input that is unreliable in terms of overall recommendations? It is hard to imagine "training" reviewers to be more reliable. Still, I believe there are steps that an editor can take to better ensure that high quality papers get published. Right now the incentive system at most major academic journals leads reviewers to view themselves as evaluators whose job is to certify the current correctness of the manuscript. At one level, this is not surprising since the process is double blind and, thus, quite impersonal. Also, encouraging a revision just means that the reviewer will have to see the paper again, adding to the person's (uncompensated) workload. Finally, since reviewers are also often authors, the process of negative reviews is self-perpetuating, i.e., why should I provide constructive reviews if I never get help from the reviewers? What is needed is to break this cycle and get reviewers to view themselves not only as evaluators, but also as mentors whose job is to help get a paper published. Such a role should not be completely alien to us, since most of us play the role of mentor or advisor for our students and fellow workers. All this leads me to believe we need to better align the incentive system for those involved in the review process to make sure that good (publishable) papers are not rejected.

\section{Possible Solutions}

When I became editor, I instituted a practice of speaking directly with the Area Editor (AE) on every paper. Often these conversations were short, because it was clear to everyone on the review team that the manuscript had an uncorrectable fatal flaw or did not yield any significant findings. Still, during this conversation, I made it my job to ask the AE how the paper could be made publishable. Interestingly, this question at times yielded a series of insightful suggestions that I was able to pass on to the author. More importantly, as a result of these suggestions, the authors were able to rethink their approach and ultimately produce a publishable paper.

In other instances, my conversations with the $\mathrm{AE}$ were much longer, often lasting an hour or more. Sometimes these conversations concerned one or more technical aspects of the paper and concentrated on the appropriateness of the approach. At other times, however, the thrust was more on how the paper could be repositioned or modified to make it more appropriate for our Marketing Science readers. In some instances, we broadened this conversation by contacting the authors directly and discussing these issues via a conference call. Said more directly, we acted as mentors.

In retrospect, I believe this change in the process was the aspect of my job as editor that I am the most proud of, since it yielded numerous constructive insights. ${ }^{1}$ However, I do not feel that I drove this mentoring mentality far enough down into the review process. I now believe that it is also necessary to get reviewers to think of themselves as mentors. This can only be done by changing the way we "measure" a reviewer.

Right now our review process is double-blind. This has many advantages, especially those concerning fairness. Still, we now publish the name of the AE on all accepted papers. Thus, not only does the AE feel some pride in knowing he/she helped guide the paper through the process, but this individual also gets some external recognition for being part of the review team.

This leads me to make the following (bold) suggestions:

(1) Reviewers should be recognized when they produce constructive reviews. One standard method would have the AEs and editor designate a set of reviewers each year who deserve special recognition and then publish their names.

(2) A second (more radical) idea is to recognize specifically the reviewers who are on successful review

\footnotetext{
${ }^{1}$ A study by Fletcher and Fletcher (1977) provides some support to my contentions that the review process can be constructive. They took original manuscripts and the revised counterparts, sent them to new reviewers, blind to the condition, and asked them to evaluate the paper. The revised manuscripts were rated higher on 33 of the 34 quality dimensions.
} 
teams, i.e., are associated with papers that are published. This recognition could be done by listing the reviewers' identity alongside the $\mathrm{AE}$ for the accepted papers or, if reviewers feel too uncomfortable with this, listing their names once per year along with all others who were on other teams that led to a publication.

(3) Faculty should modify their vitae to list not only that they were reviewers for a journal, but also that they were reviewers for specific published papers. (Most of us now list the names of students for whom we acted as mentors. One could easily argue that being a reviewer takes as much time and effort. Certainly, the impact of our advice can be as great.)

(4) The editor should keep track of each reviewer's recommendation and annually provide feedback to the editorial board using the following matrix:

Outcome of Paper

Reviewer Recommendation Accept Revise Reject

Accept

Revise

Reject

Not only will this provide useful information for the reviewer, it will also point out that the recommendations may be less reliable than one thinks.

In summary, what is needed is a way to provide new incentives to reviewers so that they are more likely to look for positive attributes of others' research. That is not to imply they should forget the role of certifying that the paper is not wrong. However, they should also step back and see how the paper could be improved enough to justify publication. Clearly, this will not be possible every time, but it should increase the volume and quality of work that gets published.

Another variation on this theme is the concept of independent versus collaborative evaluation. Most journals rely on three or four independent (unreliable) evaluations. In most instances, the editor or $\mathrm{AE}$ takes on the job of summarizing these different evaluations and providing the authors with a set of guidelines. However, this summarization is done with little or no communication between evaluators. As such, there is little learning in terms of the review team, since the information is not shared until after the decision is made.

I quickly learned that such independent processing could be improved by collaborative effort. In effect, my conversations with the AE enabled us to feed off each other's knowledge and intuition and, thus, get deeper into the paper. More technically, we increased the correlation between the error variance associated with our reviews but, I believe, greatly reduced the actual variance. The net result was better review. ${ }^{2}$

Given today's technology, it should be possible to extend this collaboration to the rest of the review team. I could envision setting up an electronic bulletin board for a particular paper that was only accessible to the review team. Reviews would still be done independently, but the reviewers would be given access to the full set of reviews once they sent their review. Then, over some limited time, e.g., a week or two, the reviewer team led by the $\mathrm{AE}$ and/or editor could build on other's knowledge and come up with a "group" decision. This combines the good aspects of independent inputs (and the competitive spirit of individuals) with the benefits of collaboration. (Of course, the final decision would still be the responsibility of the editor.)

\section{Topics and Approaches}

Besides learning a lot about the review process, I was exposed to many new ideas and topics. I do not profess to be an expert in most of these areas, nor do I believe I have any great insights as to the topics that should be explored by others. Still, being involved in so many decisions helped me crystallize my thinking about the appropriateness of certain approaches and topics. At the risk of offending some and sounding pompous to others, let me offer the following observations:

(1) Our journal needs to broaden its range of papers and topics. There still is a need to attract readers (and potential authors) who are interested in crossfunctional studies, complex strategy issues, global marketing problems, etc. One of the trademarks of our journal is that it publishes papers with sound analysis. This should continue. Still, sound analysis does not necessarily mean higher mathematics. Proving exis-

\footnotetext{
${ }^{2}$ I believe there is relevant research on group versus individual decision making that would provide support (or non-support) for this conjecture. Currently, I base my opinion on "experience."
} 
tence theorems is important and the journal should continue to publish theoretical papers. However, developing a structure that helps the reader think about a complex problem in a new way is also very valuable even though this structure is not documented with a series of equations. So is a collection of "stylized facts" that are grouped together to form a new generalization. We, as a community of marketing scientists, need to be open to a wide variety of investigations and celebrate the fact that our journal not only is "rigorous" but also is concerned with providing useful insights to tough, relevant issues. Reviewers need to be constantly reminded that rigor is only a sufficient condition.

(2) One of the key issues facing marketing scientists is implementation, yet very little is published discussing this issue. We place great weight on new theory and new methodology. This is right, since advancements in both of these areas are needed if we are to make an impact on our field. Nevertheless, neither theory nor methodology will have much impact unless it is implemented. We need to be receptive to papers that use "best practice" or maybe even "good practice" approaches (versus coming up with new approaches) to solve real world (complex) problems. Often the issue here is how to combine a series of existing methodologies or theories to yield a new (better) system or way of thinking about the problem. In other words, we need to attract and publish papers that develop a deeper understanding (theory) on how to implement our new advances and in the process help others better operate in a complex environment.

(3) Analytic models are one of the mainstays of articles published in Marketing Science. I suspect there will always be a debate about whether or not these models need to have "realistic" assumptions. Friedman (1953) makes a very strong argument that models do not have to have realistic assumptions to capture human behavior. Still, the assumed structure needs to be robust enough that one can have faith that if you modify one or more of the parameters, the results are still meaningful. Also, it seems to me that there is little managerial value in constructing a model that reproduces some set of interesting phenomena unless the model either helps one better identify and understand the underlying factors driving the solution or provides insights as to how a manager can manipulate the outcome. The former requires the model to rest on "real- istic" assumptions. The latter requires the model to be robust.

(4) Our journal has published a number of noteworthy papers that make substantial methodological advancements. This is especially true for papers concerned with analyzing scanner data. With this said, the ultimate value of these new methodologies is to provide new substantive insights and/or to solve specific managerial problems. I believe we need to encourage more work that uses these sophisticated new methodologies to provide a deeper understanding of generalizable occurrences of such things as the effects of promotions, advertising, competitive moves, etc. Papers of this type not only show the value of the methodology, they also provide new knowledge that helps others in the practice of marketing.

Finally, I would like to formally acknowledge the work of the editorial board and particularly my team of AEs. Instead of listing them all, I direct you to look at past issues of Marketing Science for their identity. In particular, I would like the AEs to know that I really valued their input and I learned a lot from our numerous conversations. Perhaps more importantly, these frequent conversations allowed me to develop a strong friendship with each one of them. They and the editorial board deserve a pat on the back from our profession for their efforts over the last four years. ${ }^{3}$

\section{References}

Akerof, G. A. 1970. The market for 'lemons': Quality uncertainty and the market mechanism. Quart. J. Econom. 84 (3) 488-500.

Campanario, J. M. 1995. On influential books and journal articles initially rejected because of negative referee's evaluations. Sci. Comm. 16 (March) 304-325.

Fletcher, R. H., S. W. Fletcher. 1997. Evidence for the effectiveness of peer review. Sci. Eng. Ethics. 3 35-50.

Friedman, M. 1953. The methodology of positive econom. Essays in Positive Economics. University of Chicago Press, Chicago. 3-43.

Gans, J. S., G. B. Shepherd. 1994. How are the mighty fallen: Rejected classic articles by leading economists. J. Econom. Perspectives. 8 (1) 165-179.

Lynch, J. 1998. Presidential address: Reviewing. J. W. Alba, J. W. Hutchinson, eds. Advances in Consumers Research, Vol. XXV. Association for Consumer Res. Provo, Utah. 1-6.

${ }^{3}$ I would like to thank Bill Boulding, Debu Purohit, and Preyas Desai for their helpful feedback. In particular, I want to acknowledge the valuable input from John Lynch, who comes to many of the same conclusions (see Lynch 1998). 